\title{
Retrospective analysis of 79 patients with orbital floor fracture: outcomes and patient-reported satisfaction
}

\author{
Olivier Senese, \\ Edward Boutremans, \\ Caroline Gossiaux, \\ Isabelle Loeb, \\ Didier Dequanter \\ Department of Maxillofacial Surgery, $\mathrm{CHU}$ \\ Saint Pierre, Université Libre de Bruxelles, \\ Brussels, Belgium
}

\begin{abstract}
Background: The aim of our retrospective study is to evaluate the management of isolated orbital floor fractures considering the clinical, functional and aesthetic results according to the surgical approach and the type of materials used.

Methods: Retrospectively, clinical, radiological, surgical, and ophthalmological data from 79 patients were collected from January 2010 to December 2016. Furthermore, included patients were interrogated on functional and aesthetic satisfaction.

Results: The main causes of trauma were physical aggression followed by accidents. The median time between trauma and surgery was 4 days. The most common surgical approaches were the subciliary and the transconjunctival ones. Alloplastic materials were used in 75 patients. In two patients, we used a combination of two grafts. Patients experienced minor immediate complications. On follow-ups, none of our patients suffered from ocular movement restrictions. Patients treated by subciliary approach had higher risk of retractile scaring compared to other surgical approaches. In our study, patients agreed to complete a questionnaire assessing functional and aesthetic outcomes with a high satisfaction score. No association between the implant material used and the results has been assessed.

Conclusion: This study describes the results of orbital floor reconstructions. Despite a variety of materials used and surgical approaches performed, we believe that the transconjunctival approach is the most suitable option with a high satisfaction score.
\end{abstract}

Keywords: Orbital floor fracture / Surgical results / Satisfaction scores

\section{INTRODUCTION}

Orbital wall fractures can either be isolated or combined with a fracture of one of the contiguous bones of the orbital rim. Concerning isolated orbital wall fractures, the most frequently fractured is the orbital floor, referred also as a "pure blowout fracture." Pure orbital blowout fracture is an entity which should be considered distinctly from other types of orbital fractures as it is more likely to be associated with soft tissue injuries resulting in functional ophthalmological complications [1]. Surgical repair

Correspondence: Didier Dequanter

Department of Maxillofacial Surgery, CHU Saint Pierre, Université Libre de Bruxelles, Rue Haute 302, Brussels 1000, Belgium

E-mail: didier.dequanter@pandora.be

Received April 17, 2018 / Revised June 7, 2018 / Accepted June 18, 2018 should be considered if diplopia, enophthalmos or limitations of oculomotor muscles movements are present, whether using an implant material or not. A wide variety of implant options exist and have been used over the years for orbital floor reconstruction, in the hope of achieving the best clinical outcome for the patient. Despite a variety of surgical options to treat orbital floor fractures, questions over operative indications, the optimal surgical timing, and the type of material needed for repair remain unresolved.

The purpose of our study is to evaluate our experiences in orbital floor reconstructive surgery with a focus on functional and aesthetic results in order to improve the quality of life of the patients. 


\section{METHODS}

Between January 2010 and December 2016, 79 patients were treated for orbital floor fracture. Patients were classified by sex, age, type of injury, clinical presentation, surgical approaches, material used and postoperative complications. Eye mobility evaluation and Hess-Lancaster test to establish the presence of diplopia were performed for all the patients by the same ophthalmologist. Patients underwent computed tomography (CT) with coronal scans. The aesthetical and functional outcome was evaluated according to the surgical approach. Moreover, patient satisfaction was subjectively assessed by a three-item questionnaire. The satisfaction rate was determinated according to a score from 0 (no satisfaction) to 5 (high satisfaction). Statistical analysis was performed using chi-square test.

\section{RESULTS}

Of the 79 patients in our study, 18 (22.8\%) were women and 61 $(77.2 \%)$ were men (male to female ratio, 2.9) with a mean age of 37 years (range, 13 to 72 years). Median time of follow-up was 30 days (range, 1 day to 42 months). Orbital wall fractures were due to physical aggression $(n=46,58 \%)$, accidents $(n=17,21 \%)$ and sports $(n=7,9 \%)$. For nine patients $(11 \%)$, the cause of trauma was unknown. Preoperative clinical findings showed that 35 patients (47\%) reported hypoesthesia of the infraorbital nerve, 15 patients (20\%) displayed enophthalmos, five patients (7\%) experienced exophthalmos. Moreover, 21 patients (28\%) showed a limitation of the eye movement and diplopia was observed in 41 patients (60\%). In our study, six patients were asymptomatic. Periorbital hematoma and/or edema occurred in almost all patients.
The clinical examination was not described in three patients and diplopia could not be assessed in two patients due to soft tissue swelling (Table 1). According to CT findings, signs of blowout fractures were present in all but two patients, for which imaging studies could not be found. Soft tissue incarceration through the bone defect were present in 18 patients (23\%); nine of which involved an oculomotor muscle. The lamina papyracea was fractured in $22(27.8 \%)$ patients. The mean time from trauma to surgery was 11 days (range, 1 to 213 days) with a median of 4 days. In 71 patients (90\%), surgical treatment was performed within the first 14 days after trauma, and in 43 patients (54.4\%) within 4 days (Fig. 1). The mean duration of surgery time was 64 minutes. The mean and the median hospital stay were both 4.1 days (from 2 to 10 days). A subciliary approach was used in the control group $(\mathrm{n}=33)$, while the transconjunctival approach was performed in $42 \%$ of the cases (experimental group). A sub-tarsal approach was used in 11 cases (17.7\%). In two patients, the surgical exposure was achieved through the traumatic wound (Fig. 2).

Seventy-six patients benefited from an implant. Thirty-four patients had a resorbable implant (Resorb-x; KLS Martin, Jacksonville, FL, USA). A permanent implant (titanium grid; Medpor Stryker, Kalamazoo, MI, USA) was used in 42 patients. Among those 76 patients, two patients had an extra bone graft. One patient had a placement of a xenograft consisting of porcine cortical bone (Lamina; OsteoBiol, Torino, Italy) and a second one had an autograft (costal graft). In three cases, the reconstructive surgery was performed without the need for an implant (4\%). Neither the type of fracture nor the type of implants used showed a significant difference in terms of clinical outcome (Tables 2, 3). On followups, none of our patients suffered from ocular movement restrictions (Table 1).

Table 1. Clinical outcomes

\begin{tabular}{lccc} 
Variable & Preoperative $(\mathrm{n}=74)$ & Immediate after surgery $(\mathrm{n}=79)$ & Postoperative 3 months $(\mathrm{n}=24)$ \\
Diplopia & $41(55)$ & $24(30)$ & $5(21)$ \\
Infraorbital hypoesthesia & $35(47)$ & $19(24)$ & $7(29)$ \\
\hline Extraocular movement limitation & $21(28)$ & $5(6)$ & 0 \\
Enophthalmos & $15(20)$ & 0 & $3(13)$ \\
\hline Exophthalmos & $5(7)$ & $2(2.5)$ & 0 \\
\hline
\end{tabular}

Values are presented as number (\%). 


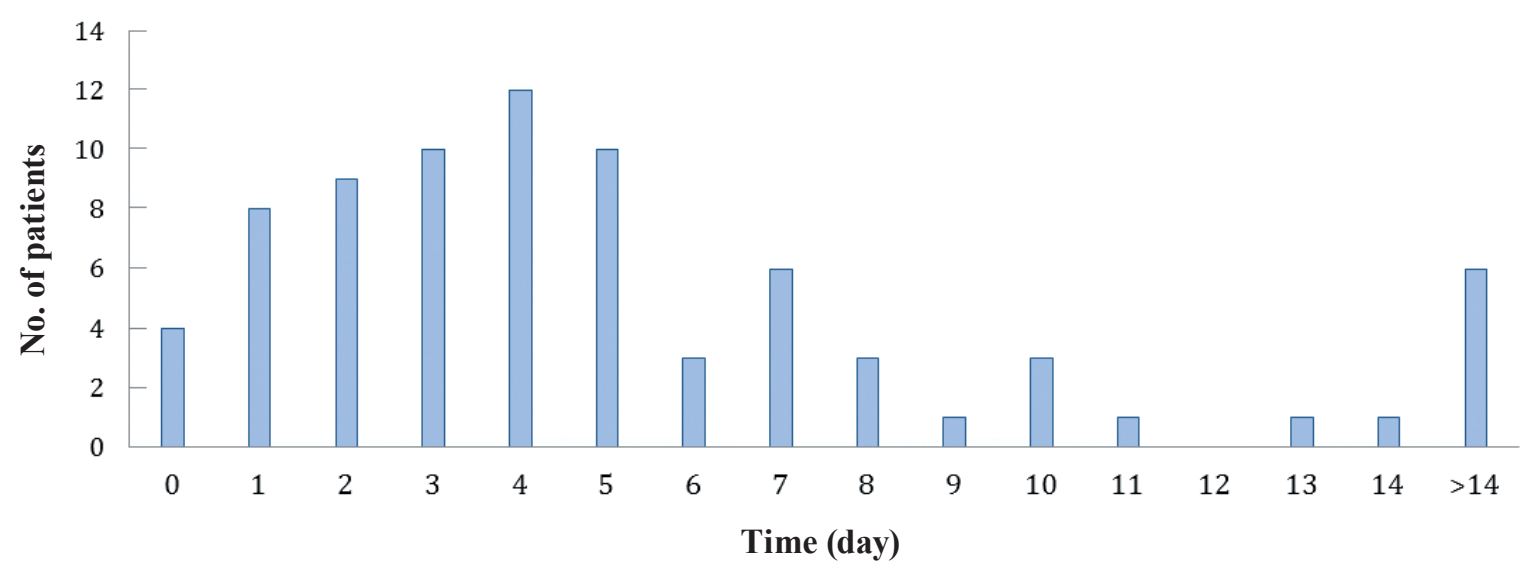

Fig. 1. Surgical timing.

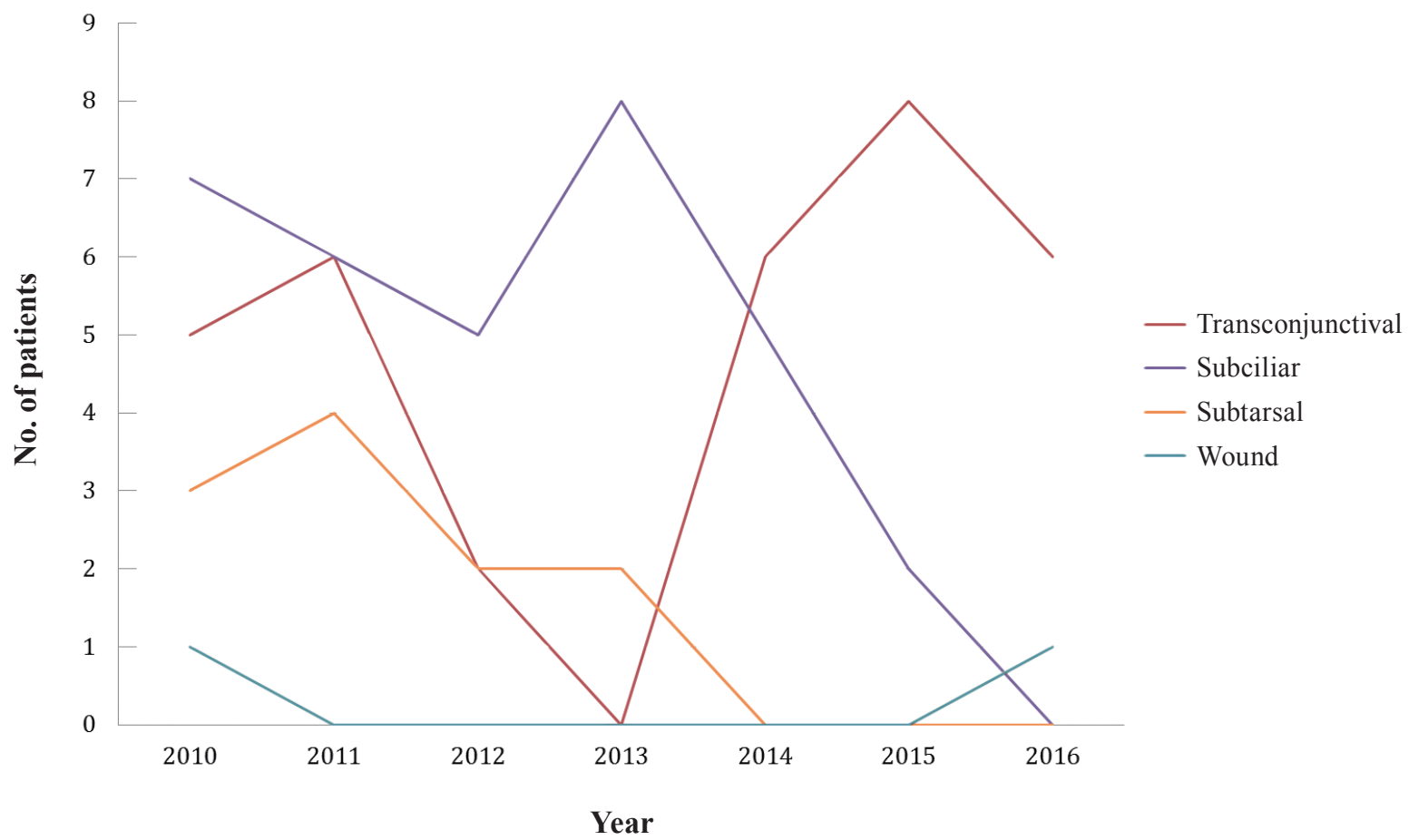

Fig. 2. Evolution of the surgical approach used during the study period.

Diplopia was observed in 24 patients (39\%) in the immediate postoperative period. Three patients (13\%) had enophthalmos 3 months after surgery. Seven patients (29\%) complained of infraorbital hypoesthesia for more than 90 days after surgery. Eight patients (10\%) experienced a retractile scar including entropion and ectropion, resolved by local treatments (Table 4). Two patients reported discomfort associated with the use of bioresorbable im- plants and, in both cases, the implants were removed. No inflammatory reaction was noted.

A second surgical procedure was necessary in two patients who presented persistent enophthalmos. Both patients were successfully treated. Furthermore, 32 of all 79 patients agreed to fill in a questionnaire on postoperative symptoms and aesthetic satisfaction. The results showed a high satisfaction score (Table 5). 
Table 2. Types of fracture

\begin{tabular}{lcccccc} 
& \multicolumn{3}{c}{ Preoperative $(n=74)$} & & \multicolumn{2}{l}{ Implant $(\mathrm{n}=71)$} \\
\cline { 2 - 6 } Variable & $\begin{array}{c}\text { Diplopia } \\
(\mathrm{n}=41)\end{array}$ & $\begin{array}{c}\text { Enophthalmos } \\
(\mathrm{n}=15)\end{array}$ & $\begin{array}{c}\text { Ocular mobility } \\
(\mathrm{n}=24)\end{array}$ & & $\begin{array}{c}\text { PLA } \\
(\mathrm{n}=30)\end{array}$ & $\begin{array}{c}\text { Titanium grid } \\
(\mathrm{n}=29)\end{array}$ \\
Orbital floor $(\mathrm{n}=52)$ & $26(50)$ & $11(21)$ & $15(29)$ & $21(40)$ & $28(54)$ \\
Orbital floor+medial wall $(\mathrm{n}=22)$ & $15(68)$ & $4(18)$ & $9(41)$ & $10(45)$ & $12(55)$ \\
\hline Statistical difference & $\chi^{2}(1)=0.76, p=0.38$ & $\chi^{2}(1)=1.36, p=0.24$ & $\chi^{2}(1)=1.28, p=0.26$ & $\chi^{2}(1)=0.01, p=0.93$ & $\chi^{2}(1)=0.01, p=0.93$ \\
\hline
\end{tabular}

Values are presented as number (\%).

PLA, poly-D, L-lactic acid.

Table 3. Type of implants and grafts

\begin{tabular}{|c|c|c|c|c|}
\hline \multirow[b]{2}{*}{ Variable } & \multicolumn{4}{|c|}{ Postoperative outcomes after 30 days $(n=29)$} \\
\hline & Diplopia & $\begin{array}{l}\text { Extraocular movement } \\
\text { limitation }\end{array}$ & Enophthalmos & $\begin{array}{l}\text { Infra orbital } \\
\text { hypoesthesia }\end{array}$ \\
\hline Total implants $(\mathrm{n}=78)$ & 11 & 2 & 5 & 11 \\
\hline Poly-D-lactic acid $(n=34)$ & 5 & 0 & 3 & 10 \\
\hline Titanium $(\mathrm{n}=42)$ & 6 & 2 & 2 & 0 \\
\hline Xenograft $(n=1)$ & 0 & 0 & 0 & 0 \\
\hline Allograft $(n=1)$ & 0 & 0 & 0 & 1 \\
\hline No implant & 0 & 0 & 0 & 0 \\
\hline
\end{tabular}

Table 4. Surgical approaches and outcome

\begin{tabular}{lcc} 
Variable & Retractile scar $<1$ month & Infraorbital hypoesthesia $>1$ month \\
Transconjunctival $(n=33)$ & $2(6)$ & $9(27)$ \\
Subciliary $(n=33)$ & $5(15)$ & $2(6)$ \\
Subtarsal $(n=11)$ & $1(9)$ & 0 \\
Wound $(n=2)$ & 0 & $1(50)$ \\
Total $(n=79)$ & $8(10)$ & $12(15)$ \\
\hline
\end{tabular}

Values are presented as number (\%).

Table 5. Satisfaction scores

\begin{tabular}{|c|c|c|c|c|c|c|}
\hline \multirow{2}{*}{ Surgical approach } & \multicolumn{6}{|c|}{ Aesthetic satisfaction } \\
\hline & 5 & 4 & 3 & 2 & 1 & Average \\
\hline Transconjunctival $(\mathrm{n}=12)$ & $10(77)$ & $2(15.3)$ & 0 & 0 & 0 & 4.8 \\
\hline Subciliary $(n=10)$ & $5(50)$ & $4(40)$ & $1(10)$ & 0 & 0 & 4.4 \\
\hline Subtarsal $(n=9)$ & $6(66)$ & $3(33)$ & 0 & 0 & 0 & 4.66 \\
\hline Wound $(n=1)$ & 0 & $1(100)$ & 0 & 0 & 0 & 4 \\
\hline Total $(n=32)$ & $21(65.6)$ & $10(31.2)$ & $1(3.1)$ & 0 & 0 & 4.63 \\
\hline
\end{tabular}

Values are presented as number (\%).

1 , no satisfaction; 2 , well; 3 , good; 4 , very good; 5 , excellent.

\section{DISCUSSION}

Surgical treatment of orbital fractures should be performed without delay [1]. In our study, the reported surgical time between the trauma and the intervention was, in $80 \%$ of cases, less than 7 days.
This attitude differs from previous studies [1]. Indeed, some authors advocated a minimum period of 7 days in order to re-evaluate the patient after disappearance of hematomas and/or edema of periorbital and intraorbital tissues [2]. Moreover, time of surgical management of facial fractures may differ from the current guide- 
lines. For example, in some cases as in the presence of intraorbital hypertension, the immediate orbital surgery may be needed to preserve patient's vision $[3,4]$.

Nowadays, controversies regarding the surgical treatment of orbital floor fractures exist and concern the type of incision and the surgical approach [5]. In our study, the subciliary approach was initially more applied but slowly, as observed in the literature $[4,5]$, these surgical approach was replaced by a transconjunctival incision to expose the orbital floor fracture. The transconjunctival approach offers a good exposure to the orbital floor without visible scar. The last years remains the use of this surgical approach stable in comparison with the subtarsal approach that is less used. The rate of complications, in our study, varied from $0 \%$ to $39 \%$, compared to $1.5 \%$ and $43.5 \%$ in literature [6].

Three complications are still frequently observed at long-term follow-up: diplopia, enophthalmos, and hypoesthesia of the infraorbital nerve territory, despite the surgical technique, a successful anatomic reconstruction and an accurate follow-up. In our series, infraorbital nerve hypoesthesia was observed in seven patients (29\%) 3 months after surgery. However, no significant difference was found between infraorbital nerve hypoesthesia and the surgical approach. In their study, Brucoli et al. [6] analyzed the incidence and the potential risk of these three complications. In their series, sex, type of fracture and implants were not significantly associated with the rate of complications $(p>0.05)$. Time interval between trauma and surgery was the only predictive factor that influenced the observed complications.

Restoration of orbital volume can prevent enophthalmos. In our series, three patients experienced enophthalmos 3 months after surgery. Actually, many different materials are proposed for reconstruction of traumatic orbital floor defects but, nowadays, no consensus exist in the literature [6,7]. In our study, 42 patients had a permanent implants. Implants made of titanium or composed with poly-D, L-lactic acid with a higher described biocompatibility [7] were mostly used. Our choice was mainly based on the size of the bone defect, on individual patient basis and surgeon's preference, factors described in the literature $[7,8]$. In our study, no difference was found between the rate of complications and the different types of materials used. However, the comparison of our results and type of implants was difficult to achieve because, especially permanent implant as the titanium grid is mostly used to restore larger defects, as reported by Schubert et al. [8]. In addition, this type of implant offers a higher rate of biocompatibility and ability to be incorporated into the soft and bone tissues. However, due to its weakness, its irregular shape and sharp edges, the use of these implant is more difficult.

Based on the literature $[9,10]$, the clinical effectiveness and safety of various bioresorbable implants in the repair of orbital fractures were evaluated. Generally, bioresorbable implants offer several advantages over permanent implants. However, Young et al. [9] found no significant difference in terms of clinical outcome independently of the type of implants and the type of fractures. In our study, two patients have benefited from bone grafts. The use of bone grafts in the reconstruction of orbital floor fractures achieved good cosmetic and functional outcomes, as reported by Zunz et al. [10]. However, after mean follow-up of 12.5 months, the perioperative or long-term complications were not significantly different.

Our study demonstrated and confirmed the variables attitudes in management of orbital floor fractures. Christensen and Zaid [11] compared the available published data concerning the management of orbital floor fractures. A consensus was found concerning the operative indications but the choice of reconstructive material and timing for surgery remain controversial. Other assessments are clearly needed. Therefore, in our series, our patients agreed to answer a questionnaire on functional and aesthetic contentedness. The treatment effectiveness was high and the reported results combined with a high satisfaction score. Our results suggest that the use of transconjunctival and subtarsal approaches increases the satisfaction score in the majority of our patients (65.6\%). Moreover, the transconjunctival approach was strongly but not significantly linked to the highest satisfaction score concerning the aesthetic results. Moreover, our results confirmed reported results that demonstrated a higher rate of retractile scars associated with the subciliary approach, and a significantly more favorable aesthetic result by performing transconjunctival and subtarsal approaches [12].

Our retrospective study evaluated 79 consecutive patients 
treated for orbital floor fractures. Although there are a wide variety of materials and surgical approaches, we believe that the transconjunctival approach is a suitable option with a high satisfaction score. Moreover, no significant association was found between the type of the implants used, the delay of surgery and the rate of complications.

\section{CONFLICT OF INTEREST}

No potential conflict of interest relevant to this article was reported.

\section{PATIENT CONSENT}

The patients provided written informed consent for the publication and the use of their images.

\section{REFERENCES}

1. Burnstine MA. Clinical recommendations for repair of isolated orbital floor fractures: an evidence-based analysis. Ophthalmology 2002;109:1207-10.

2. Poeschl PW, Baumann A, Dorner G, Russmueller G, Seemann R, Fabian F, et al. Functional outcome after surgical treatment of orbital floor fractures. Clin Oral Investig 2012;16:1297-303.
3. Pham CM, Couch SM. Oculocardiac reflex elicited by orbital floor fracture and inferior globe displacement. Am J Ophthalmol Case Rep 2017;6:4-6.

4. Gosau M, Schoneich M, Draenert FG, Ettl T, Driemel O, Reichert TE. Retrospective analysis of orbital floor fractures: complications, outcome, and review of literature. Clin Oral Investig 2011;15:305-13.

5. Liss J, Stefko ST, Chung WL. Orbital surgery: state of the art. Oral Maxillofac Surg Clin North Am 2010;22:59-71.

6. Brucoli M, Arcuri F, Cavenaghi R, Benech A. Analysis of complications after surgical repair of orbital fractures. J Craniofac Surg 2011;22:1387-90.

7. Strong EB. Orbital fractures: pathophysiology and implant materials for orbital reconstruction. Facial Plast Surg 2014;30:509-17.

8. Schubert W, Gear AJ, Lee C, Hilger PA, Haus E, Migliori MR, et al. Incorporation of titanium mesh in orbital and midface reconstruction. Plast Reconstr Surg 2002;110:1022-30.

9. Young SM, Sundar G, Lim TC, Lang SS, Thomas G, Amrith S. Use of bioresorbable implants for orbital fracture reconstruction. Br J Ophthalmol 2017;101:1080-5.

10. Zunz E, Blanc O, Leibovitch I. Traumatic orbital floor fractures: repair with autogenous bone grafts in a tertiary trauma center. J Oral Maxillofac Surg 2012;70:584-92.

11. Christensen BJ, Zaid W. Inaugural survey on practice patterns of orbital floor fractures for american oral and maxillofacial surgeons. J Oral Maxillofac Surg 2016;74:105-22.

12. Manganello-Souza LC, Rodrigues de Freitas R. Transconjunctival approach to zygomatic and orbital floor fractures. Int J Oral Maxillofac Surg 1997;26:31-4. 